TABLE V: NaTDC Molality, $m\left(\mathrm{~mol} \mathrm{~kg}^{-1}\right)$, the Relaxation Frequency, $f_{\mathrm{r}}(\mathrm{MHz})$, the Relaxation Amplitude, $10^{17} \mathrm{~A}\left(\mathrm{~cm}^{-1} \mathrm{~s}^{2}\right)$, and the Ultrasonic Absorption at Infinite Frequency, $10^{17} B\left(\mathrm{~cm}^{-1} \mathrm{~s}^{2}\right)$, of the High-Frequency Relaxation Processes

\begin{tabular}{|c|c|c|c|c|c|c|c|c|c|}
\hline \multirow[b]{2}{*}{$m$} & \multicolumn{3}{|c|}{$25^{\circ} \mathrm{C}$} & \multicolumn{3}{|c|}{$35^{\circ} \mathrm{C}$} & \multicolumn{3}{|c|}{$45^{\circ} \mathrm{C}$} \\
\hline & $f_{\mathrm{r}}$ & $10^{17} \mathrm{~A}$ & $10^{17} B$ & $\overline{f_{\mathrm{r}}}$ & $10^{17} A$ & $10^{17} B$ & $\overline{f_{\mathrm{r}}}$ & $10^{17} \mathrm{~A}$ & $10^{17} B$ \\
\hline 0.1007 & 105 & 42 & 23 & 145 & 30 & 20 & 190 & 24 & 16 \\
\hline 0.2566 & 75 & 110 & 30 & 85 & 85 & 27 & 100 & 67 & 25 \\
\hline 0.3007 & 70 & 150 & 32 & 80 & 124 & 30 & 90 & 100 & 28 \\
\hline 0.4089 & 50 & 260 & 38 & 60 & 230 & 35 & 68 & 167 & 33 \\
\hline
\end{tabular}

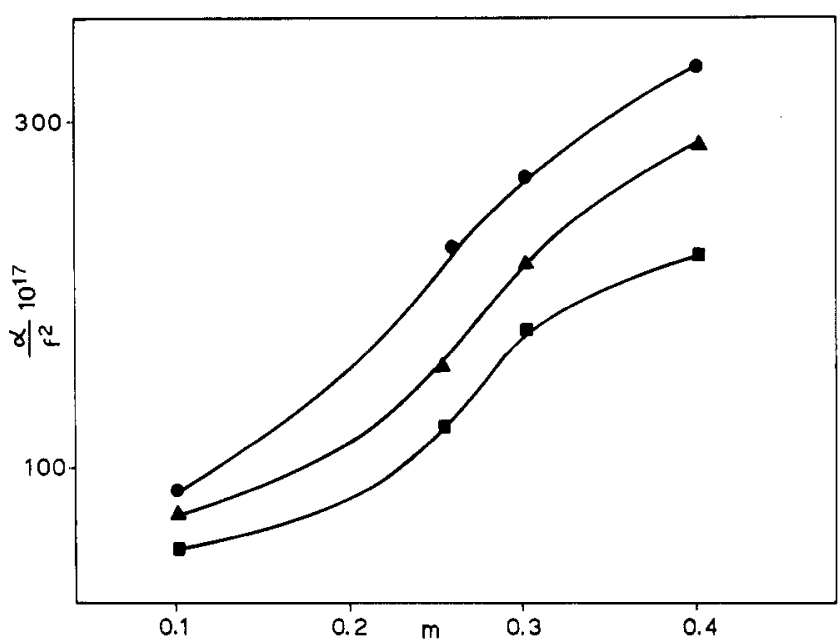

Figure 8. Dependence of the ultrasonic absorption coefficient, $\alpha / f^{2}\left(\mathrm{~cm}^{-1}\right.$ $\mathrm{s}^{2}$ ), on the NaTDC molality, $m$, at $25(\bullet) 35(\Delta)$, and $45^{\circ} \mathrm{C}(\mathbf{\square})$ at 15 MHz.

become more marked in the less concentrated solutions.

The asymmetry of the curves $\mu_{\mathrm{exc}}=\mu-\mu_{\mathrm{B}}$ vs. $\log f$, Figure 7 , is further evidence that several kinetic processes play a role in the ultrasonic absorption of these bile salt solutions.
One process, with $f_{\mathrm{r}}$ lower than $15 \mathrm{MHz}$, occurs at lower frequency with higher surfactant concentration and could be related with the change of primary micelles into flexible superaggregates via hydrogen bonds ${ }^{22}$ or via hydrophobic interactions. ${ }^{23}$

Other processes, with $f_{\mathrm{r}}$ higher than $50 \mathrm{MHz}$, should be ascribed to reactions between dispersed monomers, $\mathrm{S}$, and aggregates, $\mathrm{S}_{n}$ :

$$
\mathrm{S}+\mathrm{S}_{n} \rightleftharpoons \mathrm{S}_{n+1}
$$

An analysis, by eq 7, of the ultrasonic results in the range $50-300 \mathrm{MHz}$ gave relaxation frequencies decreasing as the surfactant concentration increases (Table V). This feature is inconsistent with bimolecular reactions 8 and could be a strong indication that several overlapping processes, with relaxation times close each other, are responsible for the ultrasonic absorption above $50 \mathrm{MHz}$.

The dependence of sound absorption on the composition of the solutions does not seem monotonic (Figure 8). For instance, at $15 \mathrm{MHz}$, plots of $\alpha / f^{2}$ against molality show an inflection at about $0.3 \mathrm{~mol} \mathrm{~kg}^{-1}$. Although the experimental points are too few to draw any quantitative conclusion, however, the phenomenon, with some differences, is evident at each temperature; moreover, it occurs in the same critical range of concentrations observed for viscometric anomalies, i.e., where rodlike micelles are supposed to exist.

The hypothesis that inflections in the functions of $\alpha / f^{2}(\mathrm{~m})$ could be related with aggregation changes of the solute was previously considered by Zana. ${ }^{24}$

Registry No. Sodium taurodeoxycholate, 1180-95-6.

(22) M. C. Carey and D. M. Small, J. Colloid Interface Sci., 31, 382 (1969).

(23) Y. Murata, G. Sugihara, N. Nishikido, and M. Tanaka, Paper present at the Symposium on Solution Behaviour of Surfactants. Theoretical and Applied Aspects, Potsdam, NY, June 30, 1980.

(24) (a) E. Graber and R. Zana, Kolloid. Z. Z. Polym., 238, 479 (1970); (b) E. Graber, J. Lang, and R. Zana, ibid., 238, 470 (1970).

\title{
Liquid Crystal Phase Transitions in a Solution of Rodlike and Disklike Particles
}

\author{
A. Stroobants and H. N. W. Lekkerkerker*
}

Vrije Universiteit Brussel, Fakulteit Wetenschappen, Eenheid Theoretische Fysische Scheikunde, Pleinlaan 2, 1050 Brussel, Belgium (Received: October 28, 1983)

The Onsager theory for the isotropic-anisotropic phase transition in a solution of rodlike particles is extended to solutions that contain a mixture of rodlike and disklike particles. Dependent on the total concentration of the particles and the fraction of rods and disks, one finds, in addition to the isotropic phase, two uniaxial nematic phases and a biaxial nematic phase.

\section{Introduction}

Zocher ${ }^{1-3}$ discovered that solutions of highly anisometric colloidal particles exceeding a critical concentration undergo an orientational order-disorder transition from an isotropic phase to an uniaxial lyotropic liquid crystal phase. Onsager ${ }^{4}$ first explained this phase transition as the result of the competition between the orientational entropy that favors disorder and the entropy effect associated with the orientation-dependent excluded volume of anisometric particles that favors order. Recently $\mathrm{Yu}$ and Saupe ${ }^{5,6}$ for the first time presented conclusive evidence for

(1) H. Zocher, Z. Anorg. Chem., 147, 91 (1925).

(2) H. Zocher and K. Jacobsohn, Kolloidchem, Beih., 28, 166 (1929).

(3) H. Zocher and W. Heller, Z. Anorg. Chem., 186, 57 (1930).

(4) L. Onsager, Ann. N.Y. Acad. Sci., 51, 627 (1949). the existence of a biaxial nematic phase. The system they studied consists of a mixture of surfactants (soap + long-chain alcohol) in water in which both rodlike and disklike micelles occur. Motivated by these experimental findings, we felt it worthwhile to extend the simple Onsager theory to solutions of rodlike and disklike particles to study the phase transitions in these systems. Dependent on the total concentration of the particles and the fraction of rods and disks a biaxial phase is indeed predicted to occur.

In section II the extension of the Onsager theory to solutions of rodlike and disklike particles is given. The predictions that follow from this theory for the concentrations, compositions, and

(5) L. J. Yu and A. Saupe, Phys. Rev. Lett., 45, 1000 (1980).

(6) A. Saupe, P. Boonbrahm, and L. J. Yu, J. Chim. Phys., 80, 7 (1983). 
order parameters of the isotropic-uniaxial and uniaxial-biaxial phase transition are presented in section III. Finally in section IV the present calculations are discussed and compared to related theories.

\section{Onsager Theory for Liquid Crystal Phase Transitions in a Solution of Rodlike and Disklike Particles}

The starting point of the Onsager theory ${ }^{4}$ for the phase separation in a solution of $N$ long hard rods of length $L$ and diameter $D(L \gg D)$ is the Helmholtz free energy of the solution in dialytic equilibrium with the solvent at temperature $T$.

$$
\begin{aligned}
& \frac{\Delta F}{N k_{\mathrm{B}} T}=\frac{F(\text { solution })-F(\text { solvent })}{N k_{\mathrm{B}} T}= \\
& \frac{\mu^{0}\left(T, \mu_{0}\right)}{k_{\mathrm{B}} T}-1+\ln c+\sigma(f)+b \cos (f)
\end{aligned}
$$

Here $\mu^{0}\left(T, \mu_{0}\right)$ represents the standard chemical potential of the particles at temperature $T$ in a solvent with chemical potential $\mu_{0}, c=N / V$ is the number density, $b=(\pi / 4) L^{2} D$ is the average excluded volume of two long rods with random orientations, and $\sigma$ and $\rho$ stand for the following functionals of the distribution function $f(\vec{a})$ of the axes $\vec{a}$ of the rodlike particles

$$
\begin{gathered}
\sigma(f)=\int f(\vec{a}) \ln 4 \pi f(\vec{a}) \mathrm{d} \Omega \\
\rho(f)=(4 / \pi) \iint \sin \gamma(\vec{a}, \vec{a}) f(\vec{a}) f(\vec{a}) \mathrm{d} \Omega \mathrm{d} \Omega^{\prime}
\end{gathered}
$$

Here $\gamma(\vec{a}, \vec{a})$ is the angle between $\vec{a}$ and $\vec{a}^{\prime}$. The free energy of the solution of thin hard disks with diameter $D$ is given by exactly the same expression as (1) with now $b=\left(\pi^{2} / 16\right) D^{3}$. Note that in the free energy (eq 1) concentration contributions of order $c^{2}$ and higher order have been neglected (second virial approximation). This approximation can be justified for long hard rods since for these systems $B_{3} / B_{2}{ }^{2} \ll 1\left(B_{2}\right.$ and $B_{3}$ are the second and third virial coefficient). As already noted by Onsager ${ }^{4}$ this is not the case for thin hard disks. Consequently, the approximation in the free energy (eq 1) will be more serious when applied to disks than to rods. This is made evident by a recent Monte Carlo study of a system of thin hard disks.

The appropriate extension of the free energy (eq 1) to a solution of $N_{1}$ long hard rods of length $L_{1}$ and diameter $D_{1}\left(L_{1} \gg D_{1}\right)$ and $N_{2}$ thin hard disks of length $L_{2}$ and diameter $D_{2}\left(D_{2} \gg L_{2}\right)$ in dialytic equilibrium with the solvent at temperature $T$ is given by

$$
\begin{aligned}
& \frac{\Delta F}{N k_{\mathrm{B}} T}= \frac{F(\text { solution })-F(\text { solvent })}{N k_{\mathrm{B}} T}= \\
& \frac{(1-x) \mu_{1}^{0}\left(T, \mu_{0}\right)+x \mu_{2}^{0}\left(T, \mu_{0}\right)}{k_{\mathrm{B}} T}-1+\ln c+ \\
&(1-x) \ln (1-x)+x \ln x+(1-x) \sigma_{1}+x \sigma_{2}+c\{(1- \\
&\left.x)^{2} b_{11} \rho_{11}+2 x(1-x) b_{12} \rho_{12}+x^{2} b_{22} \rho_{22}\right\}
\end{aligned}
$$

Here $N=N_{1}+N_{2}$ is the total number of particles, $c=N / V$ is the total number density, $(1-x)=N_{1} / N$ is the fraction of rods, $x=N_{2} / N$ is the fraction of disks, $\mu_{1}^{0}$ and $\mu_{2}{ }^{0}$ are the standard chemical potentials of the rods and the disks at temperature $T$ in a solvent with chemical potential $\mu_{0}$, and $b_{11}, b_{12}$, and $b_{22}$ are the average excluded volumes of two rods, a rod and a disk, and two disks with random orientations.

$$
\begin{gathered}
b_{11}=(\pi / 4) D_{1} L_{1}{ }^{2} \quad b_{12}=(\pi / 16) L_{1} D_{2}{ }^{2} \\
b_{22}=\left(\pi^{2} / 16\right) D_{2}{ }^{3}
\end{gathered}
$$

In the following we relate the various excluded volumes to $b_{11}$ by using the notation

$$
b_{11}=b \quad b_{12}=q b \quad b_{22}=r b
$$

(7) D. Frenkel and R. Eppenga, Phys. Rev. Lett., 49, 1089 (1982),
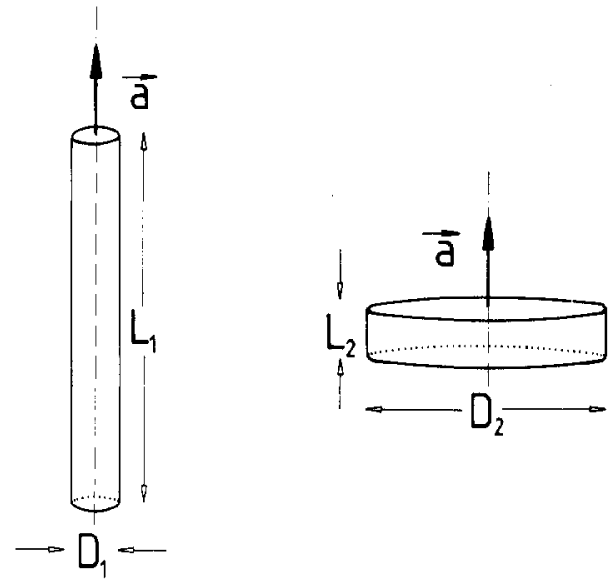

Figure 1. Orientation axes of the rodlike (a) and disklike (b) particles.

Finally, $\sigma_{j}$ and $\rho_{j k}$ stand for the following functionals of the distribution functions $f_{1}(\vec{a})$ and $f_{2}(\vec{a})$ of the axes $\vec{a}$ of the rods and disks (see Figure 1):

$$
\begin{gathered}
\sigma_{j}=\int f_{j}(\vec{a}) \ln 4 \pi f_{j}(\vec{a}) \mathrm{d} \Omega \quad j=1,2 \\
\rho_{j j}=(4 / \pi) \iint \sin \gamma(\vec{a}, \vec{a}) f_{j}(\vec{a}) f_{j}(\vec{a}) \mathrm{d} \Omega \mathrm{d} \Omega^{\prime} \quad j=1,2 \\
\rho_{12}=2 \iint|\cos \gamma(\vec{a}, \vec{a})| f_{1}(\vec{a}) f_{2}(\vec{a}) \mathrm{d} \Omega \mathrm{d} \Omega^{\prime}
\end{gathered}
$$

The orientational distribution functions $f_{1}(\vec{a})$ and $f_{2}(\vec{a})$ are determined by minimizing the free energy with respect to variations of these distribution functions. This leads to the following coupled integral equations:

$$
\begin{array}{r}
\ln 4 \pi f_{1}(\vec{a})=C_{1}-(8 / \pi) b c(1-x) \int \sin \gamma(\vec{a}, \vec{a}) f_{1}(\vec{a}) \mathrm{d} \Omega^{\prime}- \\
4 b q c x \int \mid \cos \gamma\left(\vec{a}, \vec{a}^{\prime}\right) f_{2}(\vec{a}) \mathrm{d} \Omega^{\prime}
\end{array}
$$

$\ln 4 \pi f_{2}(\vec{a})=C_{2}-4 b q c(1-x) \int|\cos \gamma(\vec{a}, \vec{a})| f_{1}(\vec{a}) \mathrm{d} \Omega^{\prime}-$

$$
(8 / \pi) b r c x \int \sin \gamma(\vec{a}, \vec{a}) f_{2}(\vec{a}) \mathrm{d} \Omega^{\prime}
$$

where $C_{1}$ and $C_{2}$ are constants that are determined by applying the appropriate normalization conditions

$$
\int f_{j}(\vec{a}) \mathrm{d} \Omega=1 \quad j=1,2
$$

Once the minimization problem has been solved, the compositions and concentrations of the coexisting phases are found by solving the coexistence conditions, i.e., the equality of the osmotic pressure $\Pi$ and the chemical potentials $\mu_{1}$ and $\mu_{2}$ of the dissolved particles in the two coexisting phases:

$$
\begin{aligned}
& \Pi=-(\partial \Delta F / \partial V)_{T, \mu_{0}, N_{1}, N_{2}}= \\
& k_{\mathrm{B}} T c\left\{1+c\left[(1-x)^{2} b \rho_{11}+2 x(1-x) b q \rho_{12}+x^{2} b r \rho_{22}\right]\right\}(12) \\
& \mu_{1}=\left(\partial \Delta F / \partial N_{1}\right)_{T: \mu_{0}, N_{2}}=\mu_{1}{ }^{0}+k_{\mathrm{B}} T \times \\
&\left\{\ln c+\ln (1-x)+\sigma_{1}+2 c\left[(1-\mathrm{x}) b \rho_{11}+x b q \rho_{12}\right]\right\}(13) \\
& \mu_{2}=\left(\partial \Delta F / \partial N_{2}\right)_{T, \mu_{0}, V, N_{1}}= \\
&\left.\mu_{2}{ }^{0}+k_{\mathrm{B}} T \ln c+\ln x+\sigma_{2}+2 c\left[(1-x) b q \rho_{12}+x b r \rho_{22}\right]\right\}
\end{aligned}
$$

At this point it is convenient to introduce dimensionless variables. In the following $c$ denotes the dimensionless concentration $b c, \Pi$ the dimensionless osmotic pressure $b \Pi /\left(k_{\mathrm{B}} T\right)$, and $\mu_{j}$ the dimensionless chemical potentials $\left.\left(\left[\mu_{j}-\mu_{j}^{0}\right) /\left(k_{\mathrm{B}} T\right)\right]+\ln b\right)$. Using these dimensionless variables and taking into account that in the isotropic phase $\sigma_{j}=0$ and $\rho_{j k}=1$ we can write the coexistence 
TABLE I: Characterization of the Isotropic and Various Liquid Crystal Phases

\begin{tabular}{|c|c|c|c|c|c|c|c|c|c|}
\hline phase & symbol & $\alpha_{11}$ & $\alpha_{12}$ & $\alpha_{21}$ & $\alpha_{22}$ & $S_{1}$ & $\Delta_{1}$ & $S_{2}$ & $\Delta_{2}$ \\
\hline isotropic & $\mathrm{i}$ & 0 & 0 & 0 & 0 & 0 & 0 & 0 & 0 \\
\hline uniaxial nematic & $n(+)$ & $>0$ & 0 & $<0$ & 0 & $>0$ & 0 & $<0$ & 0 \\
\hline uniaxial nematic & $n(-)$ & $<0$ & 0 & $>0$ & 0 & $<0$ & 0 & $>0$ & 0 \\
\hline biaxial & $\mathrm{bx}$ & $\neq 0$ & $\neq 0$ & $\neq 0$ & $\neq 0$ & $\neq 0$ & $\neq 0$ & $\neq 0$ & $\neq 0$ \\
\hline
\end{tabular}

equations between the isotropic phase (i) and an anisotropic liquid crystal phase (a) as

$$
\begin{gathered}
c_{\mathrm{i}}\left\{1+c_{\mathrm{i}}\left[\left(1-x_{\mathrm{i}}\right)^{2}+2 x_{\mathrm{i}}\left(1-x_{\mathrm{i}}\right) q+x_{\mathrm{i}}^{2} r\right]\right\}= \\
c_{\mathrm{a}}\left\{1+c_{\mathrm{a}}\left[\left(1-x_{\mathrm{a}}\right)^{2} \rho_{11}+2 x_{\mathrm{a}}\left(1-x_{\mathrm{a}}\right) q \rho_{12}+x_{\mathrm{a}}{ }^{2} r \rho_{22}\right]\right\} \\
\ln c_{\mathrm{i}}+\ln \left(1-x_{\mathrm{i}}\right)+2 c_{\mathrm{i}}\left[\left(1-x_{\mathrm{i}}\right)+x_{\mathrm{i}} q\right]= \\
\ln c_{\mathrm{a}}+\ln \left(1-x_{\mathrm{a}}\right)+\sigma_{1}+2 c_{\mathrm{a}}\left[\left(1-x_{\mathrm{a}}\right) \rho_{11}+x_{\mathrm{a}} q \rho_{12}\right] \\
\ln c_{\mathrm{i}}+\ln x_{\mathrm{i}}+2 c_{\mathrm{i}}\left[\left(1-x_{\mathrm{i}}\right) q+x_{\mathrm{i}} r\right]= \\
\ln c_{\mathrm{a}}+\ln x_{\mathrm{a}}+\sigma_{2}+2 c_{\mathrm{a}}\left[\left(1-x_{\mathrm{a}}\right) q \rho_{12}+x_{\mathrm{a}} r \rho_{22}\right]
\end{gathered}
$$

The functionals $\sigma_{j}$ and $\rho_{j k}$ are functions of $x_{\mathrm{a}}$ and $c_{\mathrm{a}}$ since through the minimization of the free energy the orientation distribution functions depend on $x_{\mathrm{a}}$ and $c_{\mathrm{a}}$. Note that in the three coexistence equations there appear four intensive thermodynamic variables, $x_{\mathrm{i}}, c_{\mathrm{i}}, x_{\mathrm{a}}$, and $c_{\mathrm{a}}$. This means that one variable can be freely chosen (in the actual calculations we will take this to be $x_{i}$ ).

\section{Evaluation of the Composition, Concentrations, and Order Parameters of the Liquid Crystal Phases}

1. Characterization of the Different Liquid Crystal Phases. A systematic way to solve the minimization problem of the free energy is to start from the coupled integral equations (eq 10) and expand the kernels $\sin \gamma$ and $|\cos \gamma|$ in Legendre polynomials. For symmetry reasons only even Legendre polynomials appear in this expansion. To simplify the calculations we limit these expansions to the $P_{2}$ term

$$
\begin{gathered}
\sin \gamma=(\pi / 4)-(5 \pi / 32) P_{2}(\cos \gamma)+\ldots \\
|\cos \gamma|=(1 / 2)+(5 / 8) P_{2}(\cos \gamma)+\ldots
\end{gathered}
$$

For the case of long rods we have shown ${ }^{8}$ that the results of calculations involving the above type of approximation are definitely quantitatively not correct but qualitatively reliable. Substituting (18) and (19) in (10) and using the addition theorem of spherical harmonics one obtains

$$
\begin{aligned}
& f_{1}(\vec{a})=\exp \left[\alpha_{11} P_{2}(\cos \theta)+\alpha_{12} D(\theta, \varphi)\right] / Z_{1} \\
& f_{2}(\vec{a})=\exp \left[\alpha_{21} P_{2}(\cos \theta)+\alpha_{22} D(\theta, \varphi)\right] / Z_{2}
\end{aligned}
$$

Here $Z_{1}$ and $Z_{2}$ are normalization constants

$$
\begin{gathered}
P_{2}(\cos \theta)=3 / 2 \cos ^{2} \theta-(1 / 2) \\
D(\theta, \varphi)=\sin ^{2} \theta \cos 2 \varphi
\end{gathered}
$$

where $\theta$ and $\varphi$ are the polar angles characterizing the orientation of the axis $\vec{a}$. The coefficients $\alpha_{j k}$ characterizing the distribution functions are determined by the consistency equations

$$
\begin{gathered}
\alpha_{11}=(5 / 4) c\left\{(1-x) S_{1}-2 x q S_{2}\right\} \\
\alpha_{12}=(15 / 16) c\left\{(1-x) \Delta_{1}-2 x q \Delta_{2}\right\} \\
\alpha_{21}=(5 / 4) c\left\{-2(1-x) q S_{1}+x r S_{2}\right\} \\
\alpha_{22}=(15 / 16) c\left\{-2(1-x) q \Delta_{1}+x r \Delta_{2}\right\}
\end{gathered}
$$

The quantities $S_{j}$ and $\Delta_{j}$ are defined as

$$
\begin{gathered}
S_{j}=\int f_{j}(\vec{a}) P_{2}(\cos \theta) \mathrm{d} \Omega=(3 / 2)\left\langle a_{z}{ }^{2}\right\rangle_{j}-(1 / 2) \\
\Delta_{j}=\int f_{j}(\vec{a}) D(\theta, \varphi) \mathrm{d} \Omega=\left\langle a_{x}{ }^{2}\right\rangle_{j}-\left\langle a_{y}{ }^{2}\right\rangle_{j}
\end{gathered}
$$

(8) H. N. W. Lekkerkerker, Ph. Coulon, R. Van Der Haegen, and R Deblieck, J. Chem. Phys, submitted for publication.

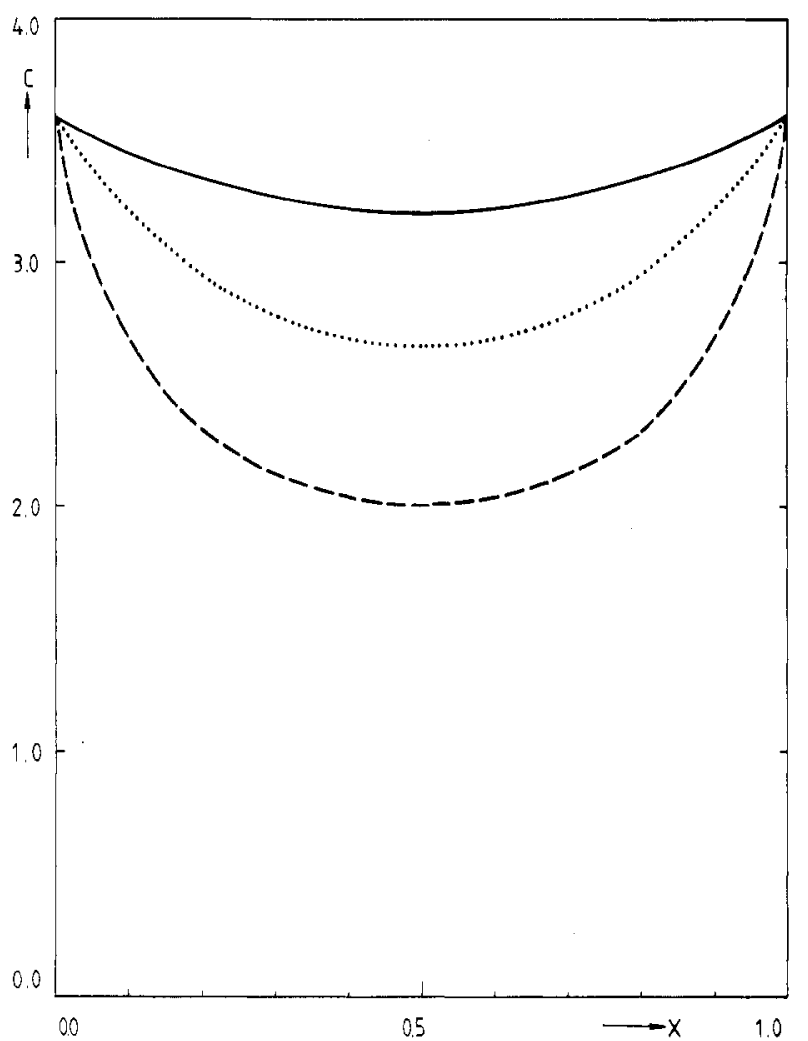

Figure 2. Lowest concentration for which a minimum appears in the free energy for a nematic phase: $(-) b_{12}=0.75 b ;(\cdots) b_{12}=b ;(---) b_{12}$ $=1.50 b$.

Using (22) and (23) and taking into account that $\vec{a}$ is an unit vector, one can write

$$
\begin{gathered}
\left\langle a_{x}{ }^{2}\right\rangle_{j}=(1 / 3)-(1 / 3) S_{j}+(1 / 2) \Delta_{j} \\
\left\langle a_{y}{ }^{2}\right\rangle_{j}=(1 / 3)-(1 / 3) S_{j}-(1 / 2) \Delta_{j} \\
\left\langle a_{z}{ }^{2}\right\rangle_{j}=(1 / 3)+(2 / 3) S_{j}
\end{gathered}
$$

The parameters $S_{1}$ and $S_{2}$ describe the ordering of the orientation of the axes of the rods and disks with respect to the $Z$ axis. The parameters $\Delta_{1}$ and $\Delta_{2}$ describe the ordering of the orientation of the axes in the $X Y$ plane. For random orientations (isotropic phase) $\left\langle a_{x}{ }^{2}\right\rangle=\left\langle a_{y}{ }^{2}\right\rangle=\left\langle a_{z}{ }^{2}\right\rangle=1 / 3$ and then $S_{1}=S_{2}=\Delta_{1}=\Delta_{2}$ $=0$. A positive value of $S_{1}$ or $S_{2}$ means that the axes of the rods or disks are oriented preferentially along the $Z$ axis whereas a negative value indicates that the axes lie preferentially in the $X Y$ plane. In the case that there is no preferred direction in the $X Y$ plane $\Delta_{1}=\Delta_{2}=0$, whereas in the case that there is a preferred direction $\Delta_{1}$ and $\Delta_{2}$ are nonzero. Solving eq 21 self-consistently for a given concentration and composition, one obtains the parameters $\alpha_{j k}$ that determine the orientation distribution functions. Note that the isotropic state $\left(S_{1}=S_{2}=\Delta_{1}=\Delta_{2}=0\right)$ represents a (trivial) solution of eq 21 that is valid for all concentrations and compositions. In the calculations pertaining to anisotropic solutions the required integrals were calculated by Gaussian integration (relative error $\leqslant 10^{-8}$ ) and the set of eq 21 was solved with an algorithme that combines the Newton method and the method of steepest descent. In the actual calculations we have limited ourselves to the simple case $b_{22}=b_{11}$, i.e., $r=1$, resulting in a phase diagram that is symmetric around $x=1 / 2$. The influence of the rod-disk excluded volume $b_{12}$ was studied by varying $q$ 
TABLE II: Composition, Concentration, Order Parameters, and Orientation-Dependent Free Energy Terms of the Coexisting Isotropic and Uniaxial Nematic Phases for $b_{11}=b_{22}=b_{12}$

\begin{tabular}{|c|c|c|c|c|c|c|c|c|c|c|}
\hline$x_{\mathbf{j}}$ & $c_{\mathrm{i}}$ & $x_{\mathrm{a}}$ & $c_{\mathrm{a}}$ & $S_{1}$ & $S_{2}$ & $\sigma_{1}$ & $\sigma_{2}$ & $\rho_{11}$ & $\rho_{12}$ & $\rho_{22}$ \\
\hline 0.000 & 3.504 & 0.000 & 3.872 & 0.574 & & 0.756 & & 0.794 & & \\
\hline 0.050 & 3.296 & 0.078 & 3.564 & 0.497 & -0.382 & 0.563 & 0.546 & 0.845 & 0.763 & 0.909 \\
\hline 0.100 & 3.144 & 0.130 & 3.320 & 0.407 & -0.344 & 0.377 & 0.407 & 0.896 & 0.825 & 0.926 \\
\hline 0.150 & 3.022 & 0.172 & 3.124 & 0.311 & -0.290 & 0.221 & 0.267 & 0.940 & 0.887 & 0.947 \\
\hline 0.200 & 2.920 & 0.212 & 2.973 & 0.222 & -0.226 & 0.114 & 0.149 & 0.969 & 0.937 & 0.968 \\
\hline 0.250 & 2.838 & 0.256 & 2.864 & 0.153 & -0.163 & 0.055 & 0.074 & 0.985 & 0.969 & 0.983 \\
\hline 0.300 & 2.773 & 0.302 & 2.786 & 0.104 & -0.113 & 0.026 & 0.034 & 0.993 & 0.985 & 0.992 \\
\hline 0.350 & 2.725 & 0.351 & 2.731 & 0.070 & -0.075 & 0.012 & 0.015 & 0.997 & 0.993 & 0.997 \\
\hline 0.400 & 2.692 & 0.400 & 2.694 & 0.043 & -0.045 & 0.005 & 0.005 & 0.999 & 0.998 & 0.999 \\
\hline 0.450 & 2.673 & 0.450 & 2.674 & 0.022 & -0.022 & 0.001 & 0.001 & 1.000 & 0.999 & 1.000 \\
\hline 0.500 & 2.667 & 0.500 & 2.667 & 0.000 & -0.000 & 0.000 & 0.000 & 1.000 & 1.000 & 1.000 \\
\hline
\end{tabular}

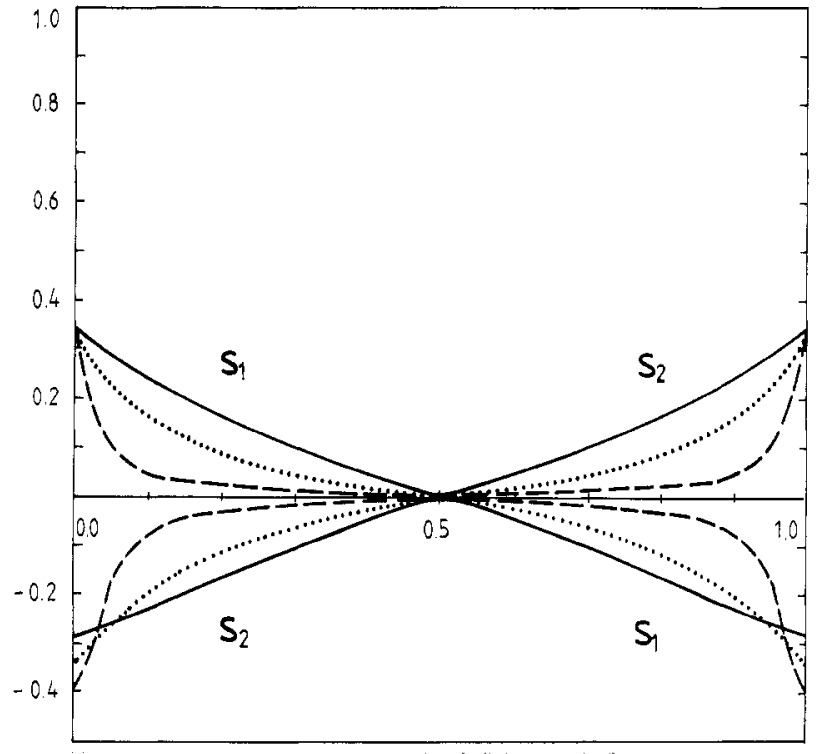

Figure 3. Values of the order parameters $S_{1}$ and $S_{2}$ for which the minimum in the free energy appears: $(-) b_{12}=0.75 b ;(\cdots) b_{12}=b ;(---)$ $b_{12}=1.50 b$.

between 0.75 and 2.0. This corresponds with ratios $L_{1} / D_{2}$ between $0.75 \pi$ and $2.0 \pi$. The coupled eq 10 give rise to three distinct types of nematic phases: two of uniaxial symmetry and one of biaxial symmetry. The distribution function coefficients and order parameters characterizing these phases are given in Table I.

2. Isotropic-Uniaxial Nematic Phase Separation. To determine the phase transition between the isotropic phase and an uniaxial nematic phase one must first minimize the free energy by solving the consistency eq 21 and subsequently solve the coexistence conditions 15-17. In Figure 2 we display for various $q$ values and as a function of composition the lowest density for which there occurs a minimum in the free energy for a $\mathrm{n}(+)$ phase $(0 \leqslant x \leqslant 1 / 2)$ and $\mathrm{n}(-)$ phase $(1 / 2 \leqslant x \leqslant 1)$. In Figure 3 we give the values of the order parameters $S_{1}$ and $S_{2}$ for which these minima occur. First of all, we see that adding disks to rods or rods to disks substantially lowers the density at which this minimum occurs. Not surprisingly this depression of the density is the stronger the larger $q$, i.e., the bigger the rod-disk excluded volume is. Secondly we note that with the exception for $x=1 / 2$ the minimum occurs for a finite value of the order parameters, i.e., we are dealing with a hard bifurcation. When $x$ approaches $1 / 2$, the jump in the order parameters becomes smaller and vanishes completely for $x=1 / 2$. This can be understood as follows. Assuming that the parameters $\alpha_{11}$ and $\alpha_{21}$ are infinitesimally small one obtains $S_{1}=\alpha_{11} / 5$ and $S_{2}=\alpha_{21} / 5$. When these values are substituted in (21) for $x=1 / 2$ it follows that the coupled equations have a solution for

$$
c=\frac{8}{1+2 q}
$$

with

$$
\alpha_{21}=-\alpha_{11}
$$

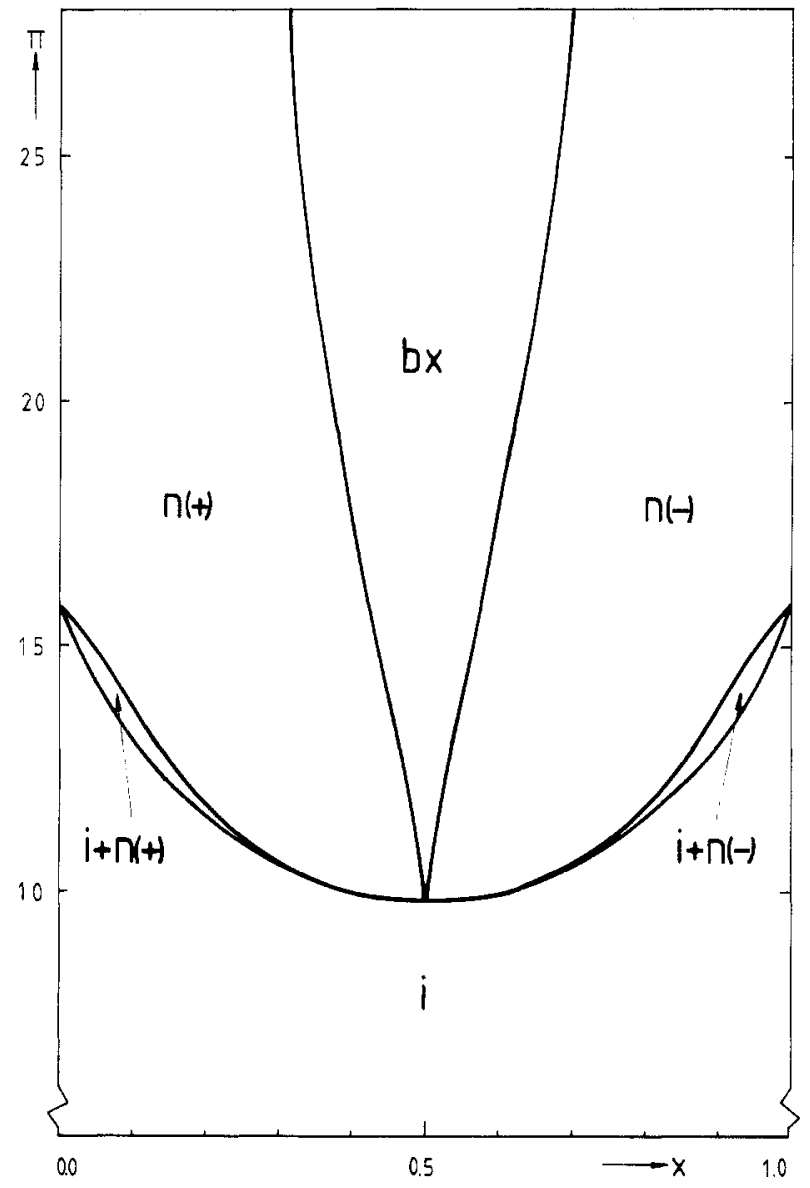

Figure 4. $\Pi-x$ diagram for the case $b_{11}=b_{22}=b_{12}$.

Expanding the free energy in $\alpha_{11}$ and $\alpha_{21}$ one finds that for $x=$ $1 / 2$ and $\alpha_{21}=-\alpha_{11}$ the cubic terms just cancel and thus the hard bifurcation becomes a continuous bifurcation.

Having established that above a certain composition-dependent minimum concentration the free energy has a minimum for a $\mathrm{n}(+)$ or $n(-)$ phase, we now consider the coexistence problem, i.e., those values of concentration and composition for which the free energy is minimized and for which coexistence conditions 15-17 are satisfied. In Table II we present the characteristic parameters of the coexisting phases for the equilibrium $\mathrm{i} \leftrightarrow \mathrm{n}(+)$ for the case $b_{11}=b_{22}=b_{12}$, i.e., $q=r=1$. The results for the equilibrium $\mathrm{i} \leftrightarrow \mathrm{n}(-)$ are, due to the fact that $b_{11}=b_{22}$, exactly the same as for the equilibrium $\mathrm{i} \leftrightarrow \mathrm{n}(+)$. One only has to replace $x_{\mathrm{i}}$ by (1 $\left.-x_{\mathrm{i}}\right)$ and $x_{\mathrm{a}}$ by $\left(1-x_{\mathrm{a}}\right)$. The $\Pi-x$ diagram for the $\mathrm{i} \leftrightarrow \mathrm{n}(+)$ and $\mathrm{i} \leftrightarrow \mathrm{n}(-)$ given in Figure 4 and the behavior of the order parameters $S_{1}$ and $S_{2}$ is presented in Figure 5. Note that the $\Pi-x$ phase diagram has the form of a minimum azeotrope.

3. Biaxial Nematic Phase. We now consider the possibility of the occurrence of a biaxial phase, i.e., a phase for which $S_{1}$ $\neq 0, S_{2} \neq 0, \Delta_{1} \neq 0, \Delta_{2} \neq 0$. We assume that the axes of the 
Liquid Crystal Phase Transitions in Solution

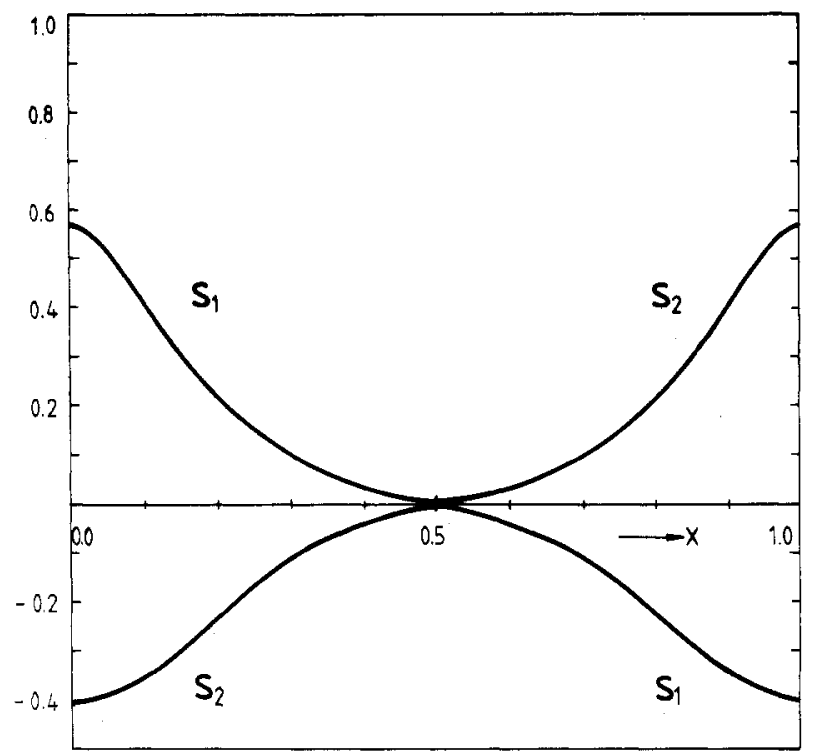

Figure 5. Order parameters $S_{1}$ and $S_{2}$ in the nematic phase that coexists with the isotropic phase for the case $b_{11}=b_{22}=b_{12}$.

rods are oriented preferentially along the $Z$ axis and the axes of the disks along the $y$ axis. Then

$$
\begin{aligned}
& \left.\left.\left\langle a_{z}{ }^{2}\right\rangle_{1}\right\rangle\left\langle a_{x}{ }^{2}\right\rangle_{1}\right\rangle\left\langle a_{y}{ }^{2}\right\rangle_{1} \\
& \left.\left.\left\langle a_{y}{ }^{2}\right\rangle_{2}\right\rangle\left\langle a_{x}{ }^{2}\right\rangle_{2}\right\rangle\left\langle a_{z}{ }^{2}\right\rangle_{2}
\end{aligned}
$$

Using (24) one find the following:

$$
S_{1}>0, \quad \Delta_{1}>0, \quad S_{2}<0, \quad \Delta_{2}<0
$$

Note that we could have made other physically equivalent choices for the preferred orientations that would have led to different signs for the order parameters. One finds that for densities above the isotropic-uniaxial nematic transition the consistency eq 21 have a solution for a biaxial phase. The free energy corresponding to this biaxial phase is lower than the free energy corresponding to the uniaxial nematic phase at the same density. The osmotic pressures for which the biaxial phase occurs are indicated in Figure 4. The behavior of the order parameters is given in Figures 6 and 7. Note that the transitions $n(+) \leftrightarrow$ bx and $n(-) \leftrightarrow$ bx are second order, i.e., the order parameters $\Delta_{1}$ and $\Delta_{2}$ characterizing the biaxiality rise from zero in a continuous way. The reason for this is that $+\Delta$ and $-\Delta$ represent the same physical state and thus the free energy is even in $\Delta_{1}$ and $\Delta_{2}$ leading to a second-order phase transition. For $x=1 / 2$ the biaxial phase arises at exactly the same concentration as that for which the transition isotropicuniaxial occurs. Again this density can be calculated analytically. Assuming distribution functions with $\alpha_{j k}$ infinitesimal one obtains $S_{1}=\alpha_{11} / 5$ and $\Delta_{1}=(4 / 15) \alpha_{12}$. Further for $b_{11}=b_{22}$ and $x=$ $1 / 2$ one has the following symmetry:

$$
\begin{aligned}
& \left\langle a_{z}{ }^{2}\right\rangle_{1}=\left\langle a_{y}{ }^{2}\right\rangle_{2} \\
& \left\langle a_{x}{ }^{2}\right\rangle_{1}=\left\langle a_{x}{ }^{2}\right\rangle_{2} \\
& \left\langle a_{y}{ }^{2}\right\rangle_{1}=\left\langle a_{z}{ }^{2}\right\rangle_{2}
\end{aligned}
$$

From (24) it then follows that

$$
\begin{gathered}
S_{2}=-(1 / 2) S_{1}-(3 / 4) \Delta_{1}=-\left(\alpha_{11} / 10\right)-\left(\alpha_{12} / 5\right) \\
\Delta_{2}=-S_{1}+(1 / 2) \Delta_{1}=-\left(\alpha_{11} / 5\right)+\left(2 \alpha_{12} / 15\right)
\end{gathered}
$$

Substituting the above values for $S_{1}, \Delta_{1}, S_{2}$, and $\Delta_{2}$ in consistency eq 21 one finds that these equations have a solution for

$$
c=\frac{8}{1+2 q}
$$

This is indeed precisely the same concentrations for which the second-order transition $\mathrm{i} \leftrightarrow \mathrm{n}(+)$ and $\mathrm{i} \leftrightarrow \mathrm{n}(-)$ occur. For $c>$
The Journal of Physical Chemistry, Vol. 88, No. 16, 1984

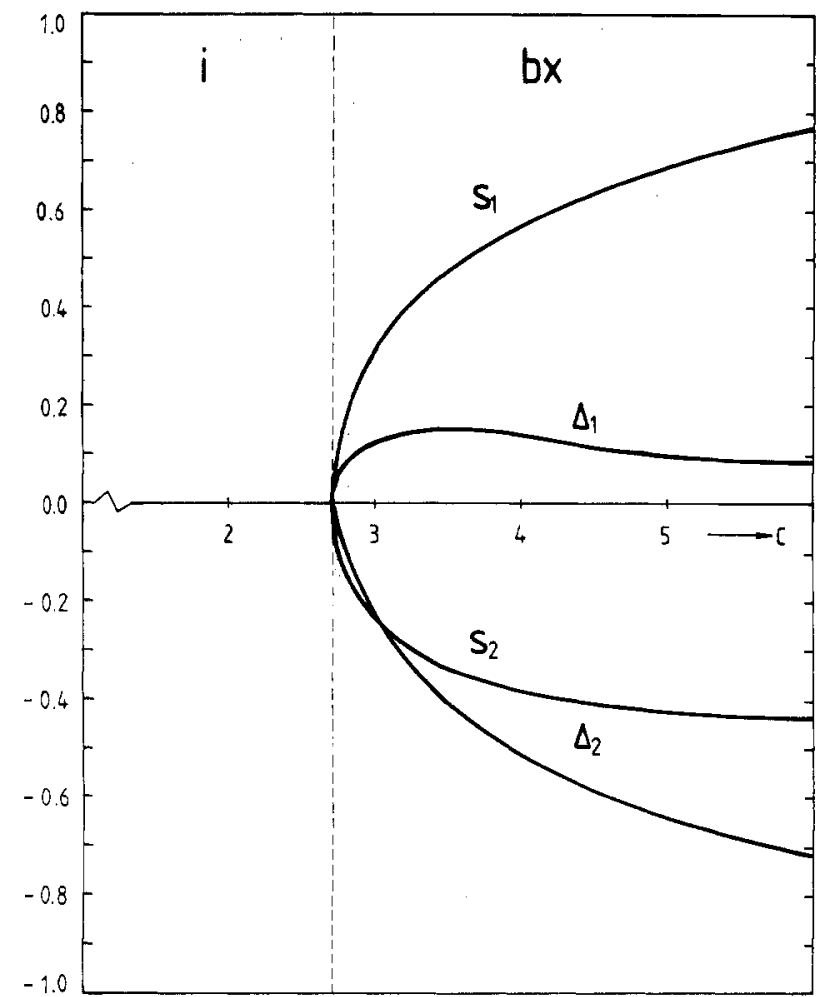

Figure 6. Order parameters $S_{1}, \Delta_{1}, S_{2}, \Delta_{2}$ in the biaxial phase for $x=$ $1 / 2$ and $b_{11}=b_{22}=b_{12}$ as a function of the concentration.

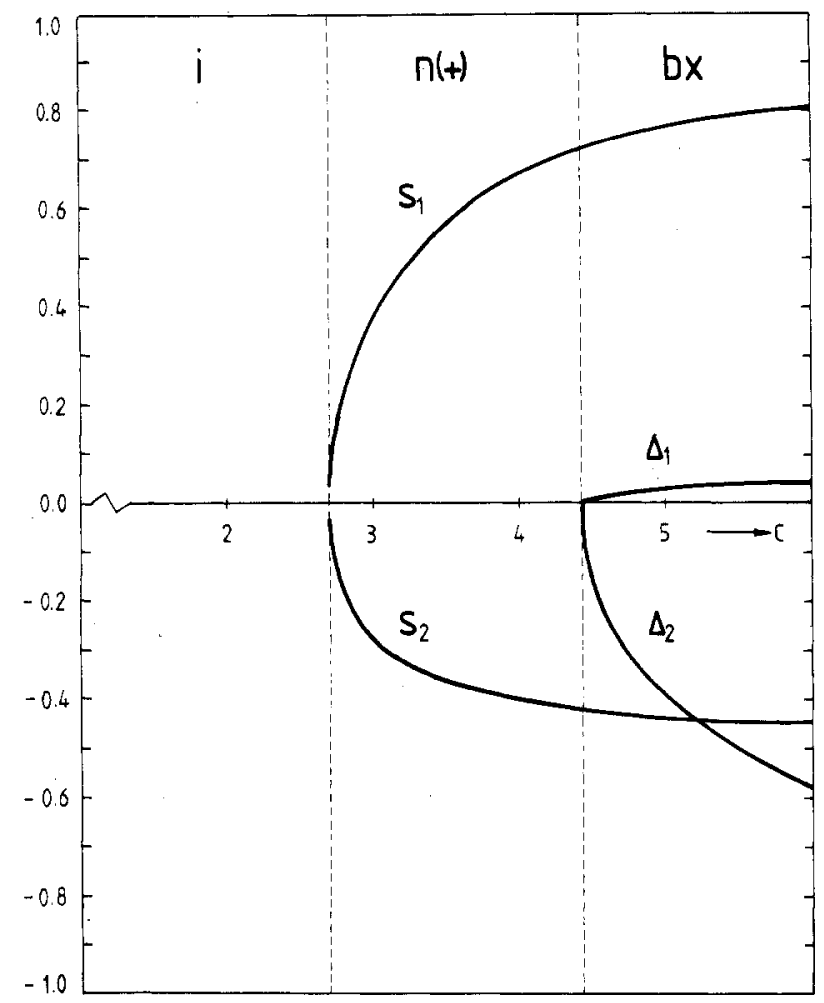

Figure 7. Order parameters $S_{1}, \Delta_{1}, S_{2}, \Delta_{2}$ in the uniaxial and biaxial phase for $x=0.4$ and $b_{11}=b_{22}=b_{12}$ as function of the concentration.

$8 /(1+2 q)$ the free energy of the biaxial phase lies lower than that of the uniaxial phases.

\section{Discussion}

In this work we have shown, using an extension of the Onsager theory, that solutions of rodlike and disklike particles depending on the concentration and composition exhibit three nematic liquid crystal phases: two of uniaxial symmetry and one of biaxial symmetry. Similar conclusions have been reached in previous 
theoretical work..$^{9,10}$ Alben $^{9}$ considered a lattice model in which only three discrete orientations are allowed $(X, Y, Z)$. This limitation is not present in the work of Rabin, McMullen and Gelbart $^{10}$ who start from a free energy that implicitly contains powers of the concentration to all orders. In their treatment, however, they pay insufficient attention to the fact that the fractions of rods and disks in the coexisting isotropic and uniaxial nematic phase are not the same. Finally, Alben ${ }^{9}$ and Saupe, Boonbrahm, and $\mathrm{Yu}^{6}$ using a Landau expansion of the free energy find a phase diagram that qualitatively shows the same features as found here.

(9) R. Alben, J. Chem. Phys., 59, 4299 (1973).

(10) Y. Rabin, W. E. McMullen, and W. M. Gelbart, Mol. Cryst. Liq. Cryst., 89, 67 (1982).
A noteworthy feature is that the biaxial phase appears in a narrow gap between the $n(+)$ and $n(-)$ phase which may explain why it has been observed in some systems ${ }^{5,6}$ but not in other quite similar systems. ${ }^{11}$ Finally, the systems where the biaxial phase has been observed ${ }^{5,6}$ have one inherent drawback: the rodlike and disklike particles are aggregates (micelles) the size, shape, and concentration of which depend on the physicochemical conditions of the system, e.g., by varying the temperature or overall surfactant concentration one also varies the characteristics of the aggregates. Therefore, it would be worthwhile to look for a biaxial phase in a solution of stable rodlike and disklike particles that keep their identity upon dilution. One might for example think of a mixture of vanadium pentoxide (rods) and clay platelets (disks).

(11) Y. Hendrickx and J. Charvolin, J. Phys., 42, 1427 (1981).

\title{
Rate Coefficients for Evaporation of Pure Liquids and Diffusion Coefficients of Vapors
}

\author{
G. Karaiskakis and N. A. Katsanos* \\ Physical Chemistry Laboratory, University of Patras, Patras, Greece (Received: November 3, 1983)
}

\begin{abstract}
The reversed-flow gas chromatography sampling technique, reported earlier, is now used to measure the diffusion coefficient of vapors from a liquid into a carrier gas and at the same time to determine the rate coefficient for the evaporation of the liquid. The mathematical expression describing the elution curves of the sample peaks is derived and used to calculate the above parameters for the liquids $n$-pentane, $n$-hexane, $n$-heptane, $n$-octane, methanol, ethanol, propan-1-ol, butan-1-ol, and pentan-1-ol evaporating into the carrier gas helium. The values of diffusion coefficients found are compared with those calculated theoretically or reported in the literature. An alternative mathematical analysis based on equilibrium at the liquid-gas interphase leads to profound disagreement with the experimental findings.
\end{abstract}

\section{Introduction}

Reversed-flow gas chromatography is a new technique, first proposed for kinetic studies in heterogeneous catalysis, ${ }^{1,2}$ applied to the dehydration of alcohols ${ }^{3}$ and the deamination of primary amines. ${ }^{4}$ The method has been extended to the determination of diffusion coefficients in binary ${ }^{5}$ and in multicomponent ${ }^{6}$ gas mixtures, the variation of these coefficients with temperature, ${ }^{7}$ as well as to other measurements like those of adsorption equilibrium constants ${ }^{8}$ and of rate constants for removal of solvents from impregnated porous solids. ${ }^{9}$ Finally, the method was used to study the heterogeneous kinetics of a complicated reaction with two gaseous reactants, namely the oxidation of carbon monoxide with oxygen over $\mathrm{Co}_{3} \mathrm{O}_{4}$-containing catalysts. ${ }^{10}$ The reversed-flow gas chromatography has been reviewed recently. ${ }^{11}$

This new method is carried out using simple gas chromatography instrumentation, and it is in reality a sampling technique based on perturbations imposed on the carrier gas flow. These perturbations consist of reversing the direction of flow of the carrier 242 .

(1) Katsanos, N. A.; Georgiadou, I, J. Chem. Soc., Chem. Commun. 1980,

(2) Katsanos, N. A. J. Chem. Soc., Faraday Trans. 1 1982, 78, 1051.

(3) Karaiskakis, G.; Katsanos, N. A.; Georgiadou, I.; Lycourghiotis, A. J.

Chem. Soc., Faraday Trans. 1 1982, 78, 2017.

(4) Kotinopoulos, M.; Karaiskakis, G.; Katsanos, N. A. J. Chem. Soc., Faraday Trans. 1 1982, 78, 3379.

(5) Katsanos, N. A.; Karaiskakis, G. J. Chromatogr. 1982, 237, 1.

(6) Karaiskakis, G.; Katsanos, N. A.; Niotis, A. Chromatographia 1983, 17,310 .

(7) Katsanos, N. A.; Karaiskakis, G. J. Chromatogr. 1983, 254, 15. 21 .

(8) Karaiskakis, G.; Katsanos, N. A.; Niotis, A. J. Chromatogr. 1982, 245,

(9) Karaiskakis, G.; Lycourghiotis, A.; Katsanos, N. A. Chromatographia 1982, 15, 351.

(10) Karaiskakis, G.; Katsanos, N. A.; Lycourghiotis, A. Can. J. Chem.

$1983,61,1853$.

(11) Katsanos, N. A.; Karaiskakis, G. Adv. Chromatogr. (N.Y.), in press. gas from time to time. Each flow reversal creates perturbations on the chromatographic elution curve, having the form of extra peaks ("sample peaks"), and thus a slow rate process, taking place within the filled or the empty chromatographic column, is sampled at known times. The analytic mathematical dependence of the heights of the sample peaks on the time when the last reversal was made is given in the Theoretical Section. This function is used to calculate the relevant rate coefficient of the slow process being sampled.

In the present work the reversed-flow technique was used to measure simultaneously the rate coefficient for the evaporation of pure liquids and the diffusion coefficient of these vapors into a carrier gas passing over the surface of the liquids at a remote point (cf. Figure 1).

The liquids used were $n$-pentane, $n$-hexane, $n$-heptane, $n$-octane, methanol, ethanol, propan-1-ol, butan-1-ol, and pentan-1-ol, the carrier gas being helium.

\section{Theoretical Section}

The general chromatographic sampling equation, describing the elution curves which follow the carrier gas flow reversals, is ${ }^{7}$

$$
\begin{gathered}
c=c_{1}\left(l^{\prime}, t_{0}+t^{\prime}+\tau\right) u(\tau)+c_{2}\left(l^{\prime}, t_{0}+t^{\prime}-\tau\right)\left[1-u\left(\tau-t^{\prime}\right)\right] \times \\
{\left[u(\tau)-u\left(\tau-t_{\mathrm{M}}\right)\right]+c_{3}\left(l^{\prime}, t_{0}-t^{\prime}+\tau\right) u\left(t_{0}+\tau-t^{\prime}\right)\left\{u\left(t-t^{\prime}\right)[1-\right.} \\
\left.\left.u\left(\tau-t_{\mathrm{M}}^{\prime}\right)\right]-u\left(\tau-t^{\prime}\right)\left[u(\tau)-u\left(\tau-t_{\mathrm{M}}\right)\right]\right\}
\end{gathered}
$$

where $c$ is the concentration of vapor at the detector, $c_{1}\left(l^{\prime}, \ldots\right)$, $c_{2}\left(l^{\prime}, \ldots\right)$, and $c_{3}\left(l^{\prime}, \ldots\right)$ are concentrations at the point $x=l^{\prime}$ ( $\mathrm{cf}$. Figure 1) for the times shown ( $t_{0}=$ total time from placing the liquid in column $L$ to the last backward reversal of gas flow, $t^{\prime}$ $=$ time interval of backward flow, $\tau=t-t_{\mathrm{M}}, t$ being the time from the last restoration of the carrier gas flow, and $t_{\mathrm{M}}$ the gas hold-up time of column section $l$ ); finally, the various $u$ 's are unit step functions for the arguments shown in parentheses and $t_{\mathrm{M}}{ }^{\prime}$ is the gas hold-up time in the section $l^{\prime}$. 